\title{
Microwave background anisotropies and large scale structure constraints on isocurvature modes in a two-field model of inflation
}

\author{
Elena Pierpaoli \\ Department of Physics and Astronomy, University of British Columbia, Vancouver, B.C. V6T 1Z1, Canada \\ Juan García-Bellido \\ Theoretical Physics, Blackett Laboratory, Imperial College, Prince Consort Road, London SW7 2BZ, U.K. \\ Stefano Borgani \\ INFN Sezione di Trieste, c/o Dipartimento di Astronomia dell'Università, via Tiepolo 11, I-34131 Trieste, Italy \\ INFN Sezione di Perugia, c/o Dipartimento di Fisica dell'Università, Via A. Pascoli, I-06123 Perugia, Italy
}

(November 17, 1999)

\begin{abstract}
In this paper we study the isocurvature mode contribution to the cosmic microwave background anisotropies and the large scale structure power spectrum, for a two-field model of inflation proposed by Linde and Mukhanov. We provide constraints on the parameters of the model by comparing its predictions with observations of the microwave background anisotropies, large scale structure data on the galaxy power spectrum, and the number density of nearby galaxy clusters. We find that such models are consistent with observations for a narrow range of parameters. As our main result, we find that only a very small isocurvature component is allowed, $\alpha \leq 0.006$, for any underlying Friedmann model. Furthermore, we give the expected resolution with which the model parameters will be determined from future satellite missions like MAP and Planck, for a fiducial flat $\Lambda$ CDM model. We find that Planck mission will be able to detect such small contributions, especially if polarization information is included. The isocurvature spectral index $n_{\text {iso }}$ will also be determined with better than $8 \%$ precision.
\end{abstract}

PACS number: 98.80.Cq Preprint IMPERIAL-TP-98/99-72, UBC-COS-99-04, hep-ph/9909420

\section{INTRODUCTION}

The future satellite experiments MAP [1] and Planck [2] open an exciting era for cosmologists and for particle physicists. The high resolution and sensitivity of these experiments will allow such a precise determination of the cosmic microwave background (CMB) power spectrum of temperature and polarization anisotropies that it will soon be conceivable to test different cosmological models with great accuracy [3]. Meanwhile, galaxy surveys, like 2dF and Sloan Digital Sky Survey (SDSS), aimed at measuring several hundred of thousand redshifts will provide a map of the Universe with unprecedented precision and extension. Any viable cosmological model must produce reasonable fits to both the observed CMB and large scale structure (LSS) power spectra, and must be tested on the basis of all available data.

Within the context of inflationary scenarios, Gaussian adiabatic fluctuations are often assumed as a standard prediction. However, besides the usual adiabatic fluctuations, other independent modes may be present, e.g. isocurvature fluctuations [- $[$ ], and in many cases they are non-Gaussian [8]. In particular, the isocurvature mode is an entropy perturbation, characterized by an appropriate balance of the fluctuations in the different components, such that the spatial curvature remains unperturbed. There is an attractive model that has been proposed some time ago that considers pure isocurvature perturbations in the baryon component [9], but unfortunately seems to be ruled out by present observations [10,11]. Most of the recent models are actually cold dark matter (CDM) isocurvature models [12], but there is also an ingenious neutrino isocurvature model [13].

Far from being academic, the reason for considering also isocurvature fluctuations resides in the fact that many different inflationary models, with more than one scalar field, predict the formation of significant isocurvature fluctuations during the inflationary era [4, 14]. As for their predictions on the density perturbation power spectra, they may differ among themselves in having different amplitudes and tilts of isocurvature and adiabatic spectra, as well as for the statistical nature of both modes. Many inflationary models predict that both the isocurvature and adiabatic fluctuations have a nearly scale invariant (Harrison-Zeldovich) spectrum with Gaussian statistics. These models have been tested against LSS in the recent literature [15,16], which showed that only a small fraction of isocurvature component seems to be allowed by present observations.

In this paper we focus on a particular inflationary scenario proposed by Linde and Mukhanov [17, in which none of the two conditions mentioned above is necessarily satisfied. In fact, here the isocurvature fluctuations are non-Gaussian, more specifically, they are $\chi^{2}$ 
distributed and their spectrum is a power law, with spectral index $n_{\text {iso }}>1$. We test this model with the present observations on CMB and LSS, and we also make predictions on how well the future satellite experiments will be able to measure the relevant model parameters.

In previous papers 15, 16,18 the mixed spectra of adiabatic plus isocurvature modes were assumed to be independent, Gaussian and approximately scale invariant. Some 15, 16 used the large scale structura data, together with the COBE-DMR normalization, to constrain the models, while others 18 only used the CMB anisotropies, with or without polarization. In our paper, we have combined both CMB and LSS observations, for a non scale invariant spectrum of perturbations, and included also the gravitational wave contribution.

In section II we review how isocurvature modes may arise from inflation, and we describe the LindeMukhanov model which inspired this work. In section III we introduce the power spectra for matter and radiation in the mixed (adiabatic + isocurvature) case, showing the effect of the amplitude and tilt of the different isocurvature contributions. In section IV] we show the comparison of the mixed spectra with the available data: we constrain the parameter range considering a joint analysis of both CMB and LSS, and we estimate with which precision the future satellite experiments will determine the relevant parameters. Finally, section $\mathrm{V}$ is dedicated to a general discussion of the results.

\section{ISOCURVATURE MODES FROM INFLATION}

Isocurvature perturbations are generated during inflation whenever there is more than one scalar field present. They correspond to entropy perturbations that do not perturb the metric, and thus the spatial curvature. They typically arise when one of the fields is fixed by its potential during inflation, the inflaton energy later decays into relativistic particles and redshifts away, while the other field's energy becomes the dominant contribution. Depending on model parameters, the relative contribution of adiabatic to isocurvature perturbations may be noticeable in the microwave background anisotropies and large scale structure, and they may have in principle very different spectral tilts, e.g. blue $(n>1)$ or red $(n<1)$. Furthermore, depending on the evolution during inflation, the statistics of the different components (adiabatic and isocurvature) could be very different, e.g. Gaussian and $\chi^{2}$ distributed, respectively. Such a complicated phenomenology requires a detail analysis in order to confirm whether a particular model is ruled out by observations.

\footnotetext{
${ }^{1}$ However, slight variants of the model can also give a Gaussian isocurvature mode [17].
}

In this paper we will concentrate in a particular realization of a mixed adiabatic and isocurvature model proposed recently by Linde and Mukhanov.

\section{A. The Linde-Mukhanov model}

The model of Ref. [17] is probably the simplest one can think of that produces isocurvature perturbations during inflation. It has two coupled massive scalar fields described by the scalar potential,

$$
V(\phi, \sigma)=\frac{1}{2} M^{2} \phi^{2}+\frac{1}{2} m^{2} \sigma^{2}+\frac{1}{2} g^{2} \sigma^{2} \phi^{2} .
$$

In principle, inflation could occur along either of the valleys at $\phi=0$ or $\sigma=0$, depending on initial conditions. In a chaotic inflation approach one expects that the fields will start at very large values, $\phi, \sigma \gg M_{P}$, where the coupling term $g^{2} \sigma^{2} \phi^{2}$ dominates. Let us suppose that initially one of the fields has a larger value, say $|\phi|>|\sigma|$ and thus the field $\sigma$ rapidly settles at $\sigma=0$. Then inflation occurs along the $\sigma=0$ valley, with energy density $\frac{1}{2} M^{2} \phi^{2}$ and a Hubble constant

$$
H^{2}=\frac{4 \pi M^{2}}{3 M_{P}^{2}} \phi^{2} .
$$

During inflation the mass of the $\sigma$ field becomes

$$
\bar{m}^{2}=m^{2}+\nu H^{2}
$$

where $\nu=3 g^{2} M_{P}^{2} / 4 \pi M^{2}$ is a constant. Typically, during inflation the second term dominates and thus the model gives a mass term of the $\sigma$ field proportional to the rate of expansion.

The quasi-de-Sitter evolution during inflation provides a neat way to generate metric perturbations from quantum fluctuations. Those of the inflaton will give rise to adiabatic density perturbations, since the energy density during inflation is proportional to the inflaton field fluctuations, $\delta \rho \sim V^{\prime}(\phi) \delta \phi$. On the other hand, quantum fluctuations of the $\sigma$ field will not generate curvature perturbations since the inflationary trajectory lies along $\langle\sigma\rangle=0$. Nevertheless, after inflation the energy density of the $\sigma$ field may come to dominate the evolution of the universe (e.g. as a cold dark matter component) and its fluctuations would then contribute as isocurvature perturbations [4]. Let us compute the amplitude of those perturbations. For a massive field with $\bar{m}^{2} \ll H^{2}$ during inflation, the amplitude of the long wavelength perturbation of the $\sigma$ field at the end of inflation is given by

$$
k^{3}\left|\sigma_{k}^{2}\right|=\frac{H^{2}}{2}\left(\frac{k}{H}\right)^{2 \bar{m}^{2} / 3 H^{2}}
$$

and the average perturbations of energy density in the $\sigma$ field, $\delta \rho_{\sigma}=\bar{m}^{2}(\delta \sigma)^{2} / 2$ can be estimated as [17] 


$$
\frac{\delta \rho_{\sigma}}{\rho_{\mathrm{tot}}} \sim \frac{\bar{m}^{2}}{M_{P}^{2}}\left(\frac{k}{H}\right)^{2 \bar{m}^{2} / 3 H^{2}},
$$

which corresponds to a "blue" spectral index

$$
n_{\text {iso }} \approx 1+4 \bar{m}^{2} / 3 H^{2} \simeq 1+4 \nu / 3>1 .
$$

The small ratio $\bar{m}^{2} / M_{P}^{2}$ ensures that the $\sigma$ field does not contribute initially to the perturbations of the metric, i.e. it generates isocurvature perturbations, which much later could end up dominating, as mentioned above. The detailed evolution is very model dependent [17. We will assume, following Linde and Mukhanov, that the correction $\nu H^{2}$ to the mass of the $\sigma$ field disappears soon after inflation, when the inflaton field $\phi$ decays into relativistic particles while the $\sigma$ field remains stable or decays very late, and thus its energy density (in coherent oscillations of the field) may dominate in the form of cold dark matter today. Under these assumptions one can estimate the corresponding density contrast [17]

$$
\frac{\delta \rho_{\sigma}}{\rho_{\sigma}} \sim C(k)\left(\frac{k}{M}\right)^{2 \nu / 3},
$$

for $k \ll \min [M \sqrt{m / M}, M \exp (-1 / 2 \nu)]$, where

$$
C(k)=\frac{\sqrt{\nu}}{\ln (M / m)+3 / \nu}\left[\ln \left(\frac{M}{k}\right)\right]^{1-\nu / 3}\left(\frac{M}{m}\right)^{2 \nu / 3} .
$$

This isocurvature perturbation has non-Gaussian statistics, in fact a $\chi^{2}$ distribution, because it arises from the square of a Gaussian field [see Eq. (5)]. 2

On the other hand, the adiabatic density perturbations generated by the inflaton field $\phi$ during inflation contribute with a Gaussian spectrum with amplitude

$$
\frac{\delta \rho_{k}}{\rho} \simeq \sqrt{\frac{4}{3 \pi}} N_{k} \frac{M}{M_{P}}\left(\frac{k}{a H}\right)^{-2 / N_{k}},
$$

where $N_{k}=N_{\text {hor }}=65$ is the number of e-folds before the end of inflation when the mode with wavenumber $k$ corresponding to our present horizon crossed the Hubble scale during inflation, with the spectral index

$$
n_{\text {ad }}=1-\frac{2}{N_{k}} .
$$

There is enough freedom in the model parameters $(\nu, M, m)$ to have the Gaussian adiabatic perturbations

\footnotetext{
${ }^{2}$ One could avoid having non-Gaussian statistics for the isocurvature component by giving the $\sigma$ field a vacuum expectation value, $\langle\sigma\rangle \neq 0$. In this way, the main isocurvature contribution would come from the term $\delta \rho_{\sigma}=\bar{m}^{2}\langle\sigma\rangle \delta \sigma$, which is Gaussian distributed.
}

dominate the spectrum on large scales, while the nonGaussian isocurvature perturbations dominate on small scales. In practice, the latter will never dominate at scales of cosmological interest since, as we shall illustrate in Sections IV and V, the relative contribution of the isocurvature component is constrained by present observations to be very small.

We will thus consider a mixed model of adiabatic and isocurvature perturbations arising from inflation and contributing simultaneously to the temperature anisotropies of the microwave background and the power spectrum of large scale structure. The relative tilts and amplitudes of the two spectra will allow us to compare with present observations and determine the likelihood functions for a given set of model parameters.

We will use the CMBFAST code [19,20] to normalize the model to COBE data 21 23, compute the $C_{l}$ and power spectra $P(k)$, and then compare with observations.

Therefore, the post-inflationary power spectra for the independent adiabatic and isocurvature components read

$$
\begin{gathered}
P_{\text {ad }}(k)=25 \times 10^{-10}\left(\frac{k}{k_{\text {hor }}}\right)^{n_{\text {ad }}}, \\
P_{\text {iso }}(k)=d_{10} \times 10^{-10}\left(\frac{k}{k_{\text {gal }}}\right)^{n_{\text {iso }}},
\end{gathered}
$$

where $d_{10}=\mathcal{O}(1-20)$ is an arbitrary normalization factor, to be fixed by observations. We will discuss in the next Section the relative normalization of the two components for a mixed power spectrum.

\section{MATTER AND RADIATION POWER SPECTRA}

In order to compare the theory with the data, we compute the power spectra of matter and radiation at the present epoch. For this purpose, we solve Boltzmann equations using CMBFAST, starting from a very early epoch $\left(z \simeq 10^{7}\right)$ up to the present time.

In the presence of only one mode of fluctuations the actual matter power spectrum is:

$$
P(k)=T^{2}(k) P_{\text {in }}(k),
$$

where $P_{\text {in }}(k)$ denotes the initial power spectrum and the transfer function $T(k)$ contains the information on how the spectrum is modified through the evolution. $T(k)$ is typically of order 1 on large scales (small $k$ ) and it becomes less than 1 at smaller scales (larger $k$ ), the actual shape depending on the specific mode considered (i.e., whether adiabatic or isocurvature), the cosmological parameters and on the nature of the dark matter content. In our analysis, while we will allow for both adiabatic and isocurvature fluctuations for general Friedmann background, we will assume the dark matter content to be contributed only by CDM and baryons, ignoring any 


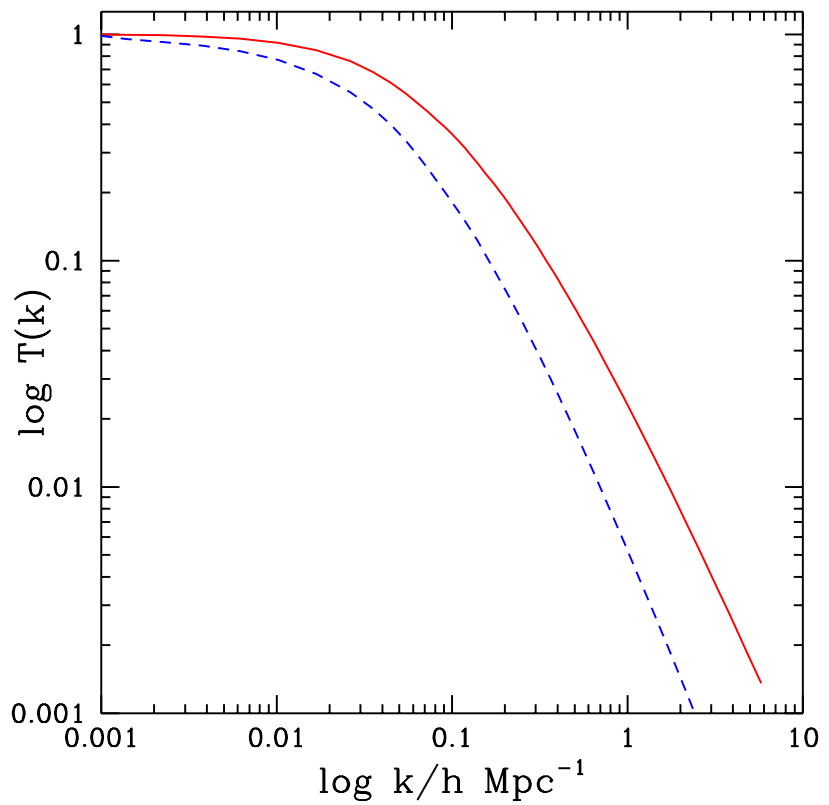

FIG. 1. Transfer function for the adiabatic (solid curve) and isocurvature (dashed curve) modes in the standard cold dark matter model $\left(\Omega_{m}=1, h=0.5\right.$ and $\left.\Omega_{\mathrm{B}}=0.05\right)$. The isocurvature mode is more damped on small scales.

contribution that may come from neutrinos in the form of hot dark matter.

As for the adiabatic and isocurvature transfer functions, we take the CDM expressions

$$
\begin{aligned}
& T_{\text {ad }}(k)=\frac{\ln (1+2.34 q)}{2.34 q} \times \\
& {\left[1+3.89 q+(16.1 q)^{2}+(5.46 q)^{3}+(6.71 q)^{4}\right]^{-1 / 4} ;} \\
& T_{\text {iso }}(k)= \\
& {\left[1+\frac{(40 q)^{2}}{1+215 q+(16 q)^{2}(1+0.5 q)^{-1}}+(5.6 q)^{8 / 5}\right]^{-5 / 4}}
\end{aligned}
$$

as provided by Bardeen et al. 24, where $q=k / \Gamma$ $\left(h^{-1} \mathrm{Mpc}\right)^{-1}$. Here $\Gamma$ is the so called "shape" parameter and for CDM model is defined as $\Gamma=h \Omega_{m} \exp \left(-\Omega_{\mathrm{B}}-\right.$ $\left.\sqrt{2 h} \Omega_{\mathrm{B}} / \Omega_{m}\right)$, so as to account for the small-scale damping due to the presence of a non-negligible baryon fraction [25. Note that we have redefined Eq. (15) with respect to the expression given in Ref. [24] so as to have the same small- $k$ asymptotic behavior, $T(k) \rightarrow 1$, for both components. Among other things, this means that $n_{\text {iso }}=1$ corresponds to a scale invariant spectrum, instead of the usual $n_{\text {iso }}=-3$ appearing in the literature. We verified that Eqs. (14) and (15) reproduce quite well the outputs of the CMBFAST code for the $k$-range of interest for our analysis.

As an example, we show in Figure 1 the shape of these two transfer functions for a particular choice of the cosmological parameters. It is apparent that the isocurva-

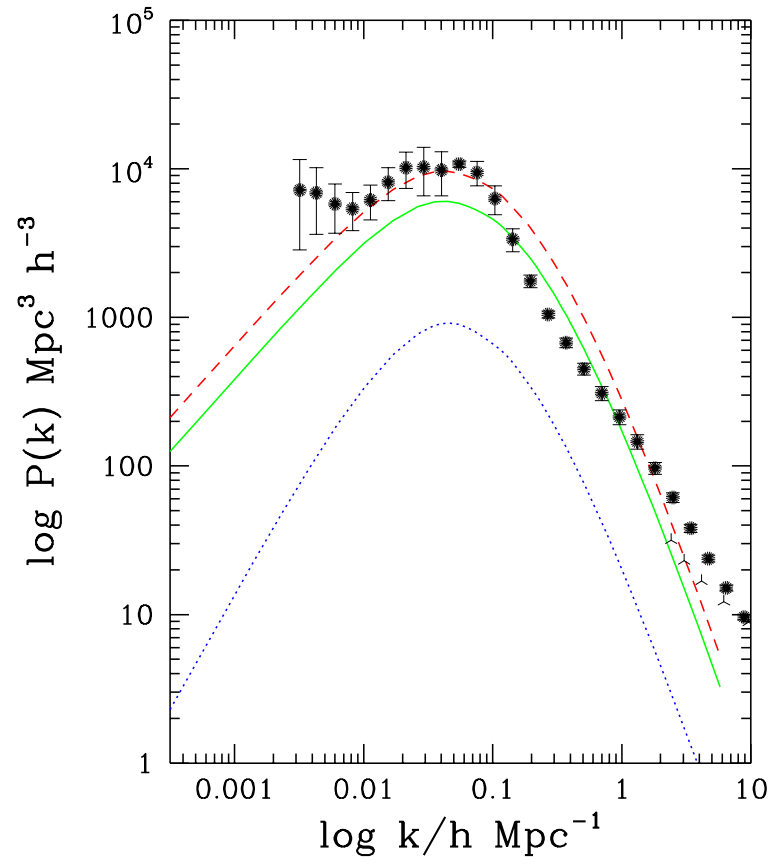

FIG. 2. Power spectra of pure isocurvature (dotted curve) and adiabatic (dashed curve) modes with spectral indices $n_{\text {ad }}=0.962$ and $n_{\text {iso }}=1.56$. The middle (solid curve) power spectrum is a mixed one, corresponding to $\alpha=8 \times 10^{-3}$. The data points are from the APM survey.

ture case has a larger damping at large $k$ values, due to the fact that they start out as zero-curvature or isothermal fluctuations and takes longer than the adiabatic ones to build up, after the matter-radiation equality epoch.

Since adiabatic and isocurvature are independent modes of fluctuations, when both modes are present at the same time the total power spectra of matter and radiation can be computed as a linear combination of the pure isocurvature and adiabatic ones. In the LM model considered in this work, both the adiabatic and isocurvature modes have power law initial power spectra, but with different spectral indices. Therefore, the overall spectrum can be casted in the form

$$
P_{\text {mix }}(k)=N_{\text {mix }}\left[\left(k \tau_{0}\right)^{n_{\text {ad }}} T_{\text {ad }}^{2}(k)+\alpha\left(k \tau_{0}\right)^{n_{\text {iso }}} T_{\text {iso }}^{2}(k)\right],
$$

where $\tau_{0}$ is the conformal time at present and $\alpha$ is a dimensionless parameter that indicates the relative contribution of isocurvature and adiabatic perturbations (pure adiabatic and isocurvature power spectra correspond to $\alpha=0$ and $\alpha=\infty$ respectively). The comparison of our $\alpha$ with the mixing coefficients introduced by other authors (e.g. 15, 16, 18]) may not be straightforward because, in contrast with their approach, we have also allowed for $n_{\text {ad }} \neq n_{\text {iso }} \neq 1$. In the case $n_{\text {ad }}=n_{\text {iso }}=1$, our $\alpha$ compares with the other choices as follows: $\alpha=\left(1-\alpha_{S}\right) / \alpha_{S}$ [15], $\alpha=\alpha_{K K S Y}$ [16], $\alpha=\alpha_{E K} /\left(1-\alpha_{E K}\right)$ [18]. In 


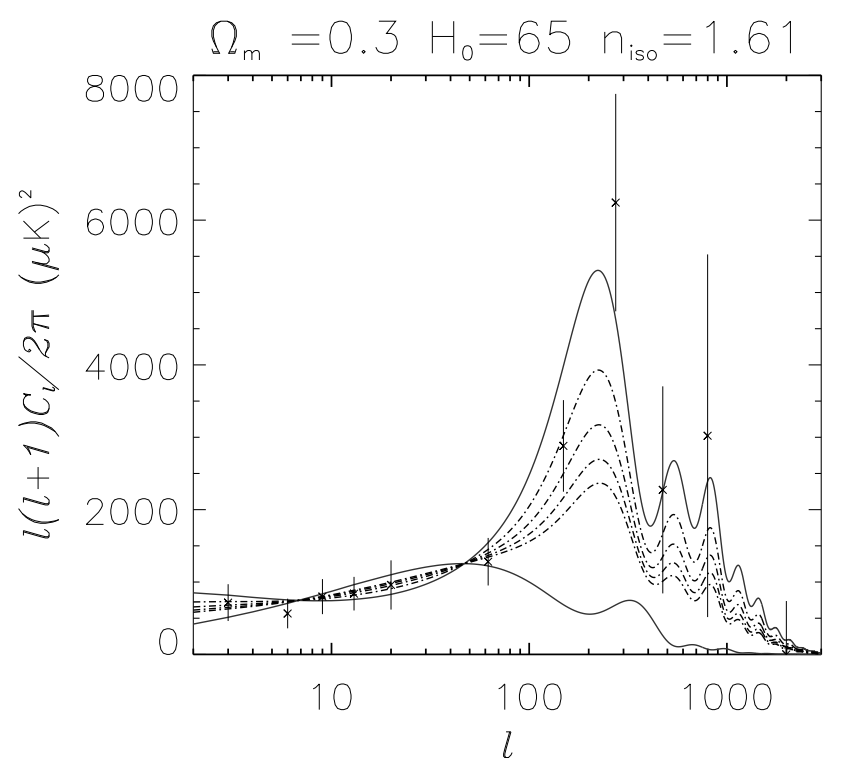

FIG. 3. Mixed CMB spectra with fixed $n_{\text {iso }}$ value. The solid lines correspond to the pure adiabatic and isocurvature modes, normalized to COBE. The dotted-dashed lines corresponds to $\alpha=3,6,9,12 \times 10^{-3}$ from top to bottom. The points shown here are the binned spectrum from Ref. [27]. However, we have used the whole set of experiments from Table fin the CMB analysis.

eq.(16), $N_{\text {mix }}$ is the normalization factor, that we derived by normalizing the $\mathrm{CMB}$ spectrum to the $\mathrm{COBE}$ data [22,23]. Different mixing coefficients $\alpha$ lead to different normalizations:

$$
N_{\text {mix }}=\frac{N_{\text {ad }}}{1+\alpha f} .
$$

In the above expression, $f=N_{\text {ad }} / N_{\text {iso }}$ where $N_{\text {ad }}$ and $N_{\text {iso }}$ are the normalizations of the pure adiabatic and isocurvature scalar modes, and their ratio conveys the information about the different Sachs-Wolfe contribution of isocurvature and adiabatic modes to $\mathrm{CMB}$ anisotropies. In the normalization, we also took into account the tensor contribution to the anisotropies, according to the parameter specified below. A typical hybrid spectrum is plotted in fig. 2, together with the APM data points [26]. Given the big tilt of the isocurvature power spectrum, the mixed one has a slope similar to the adiabatic one at small $k$. The effect of the different normalization is evident even for low values of the $\alpha$ coefficient.

As for the CMB anisotropies, following the standard notation, we describe their dependence on the direction $\hat{n}$ as

$$
\frac{\Delta T(\hat{n})}{T_{o}}=\sum_{l=0}^{\infty} \sum_{m=-l}^{l} a_{l m} Y_{l m}(\hat{n}) .
$$

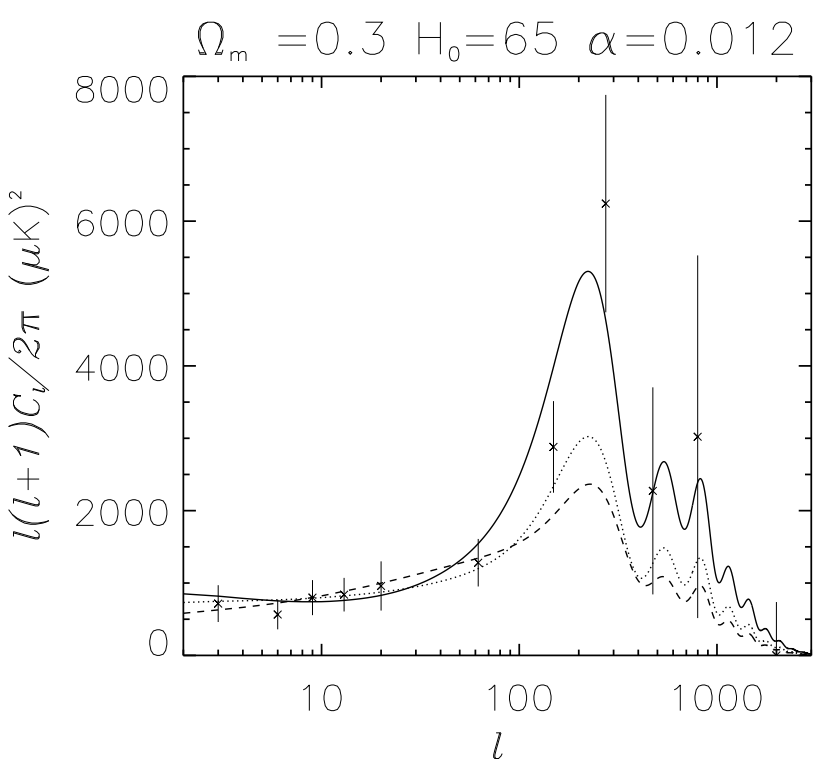

FIG. 4. Mixed $C_{l}$ spectra with different tilts of the isocurvature component with fixed ratio $\alpha=0.012$. The solid line is the pure adiabatic mode $\left(n_{\text {ad }}=0.962\right)$, the dotted line corresponds to mixed isocurvature $(\alpha=0.012)$ with $n_{\text {iso }}=1.36$ and the dashed line to $n_{\text {iso }}=1.61$.

Therefore, the radiation power spectrum is defined as

$$
C_{l}=\left\langle\left|a_{l m}\right|^{2}\right\rangle,
$$

where the brackets denote the ensemble average over different realizations. When both scalar and tensor modes are present, the $C_{l}$ can be decomposed as $C_{l}=C_{l}^{S}+C_{l}^{T}$. In Linde-Mukhanov model the tensor contribution is included in the adiabatic mode.

Similarly to what happens to the matter power spectrum, the radiation power spectrum for the mixed case can be found as a linear combination of the two independent adiabatic and isocurvature power spectra $C_{l}^{\text {ad }}$ and $C_{l}^{\text {iso }}$ :

$$
C_{l}^{\mathrm{mix}}=\frac{C_{l}^{\mathrm{ad}}+f \alpha C_{l}^{\mathrm{iso}}}{1+f \alpha} .
$$

In the expression above $C_{l}^{\text {ad }}$ and $C_{l}^{\text {iso }}$ are both normalized to $\mathrm{COBE}$, according to ref. [23]. In computing the power spectra, we modified the CMBFAST code 19,20 to our purposes. In figures 3 and 14 we show some examples of CMB spectra, together with the estimated binned spectrum from Bond et al. [27], which provides a visual indication of the experimental status. However, we have used the whole set of experiments from Table if in the CMB analysis. The effect of adding an isocurvature component is to add a lot of power on small multipoles through the SW effect. The anti-tilt of the spectrum is in general not enough to compensate this effect, and the first 


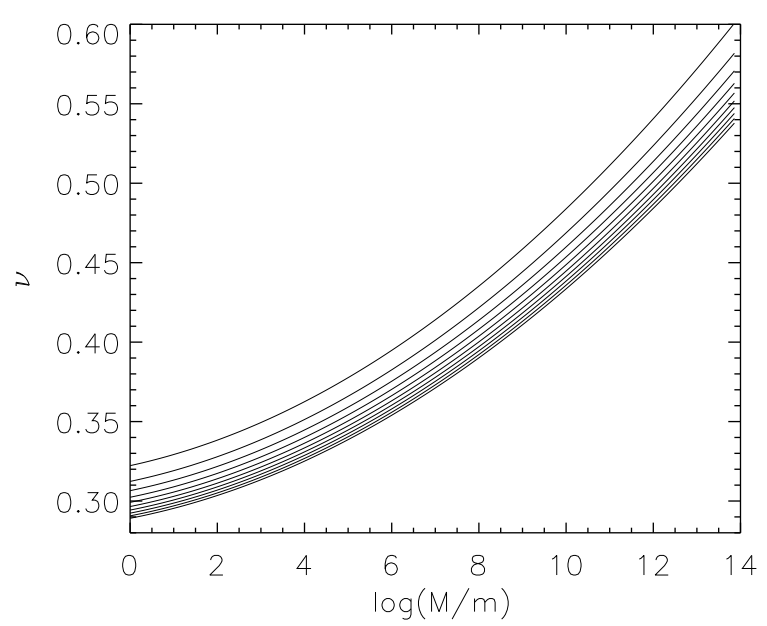

FIG. 5. Typical values of $\nu$ as a function of $M / m$, with $d_{10}$ in the range $1-10$, from top to bottom.

acoustic peak is consequently lower than in the corresponding pure adiabatic case. Fig. 3 shows examples of mixed spectra with different $\alpha$ values for fixed $n_{\text {iso }}$ and $n_{\text {ad }}$. It shows that even a small $\alpha$ can cause a significant damping of the acoustic peaks. Moreover note that a high $n_{\text {iso }}$ component also leads to suppressed acoustic peaks, for a fixed $\alpha$ value (see Fig. (4). This is because the bigger is $n_{\text {iso }}$ the smaller is the normalization coefficient $N_{\text {iso }}$ and the bigger is $f$. Therefore for fixed $\alpha$, the isocurvature $C_{l}$ spectrum takes more weight in the mixture.

\section{A. Choice of model parameters}

We will consider here a set of values for the parameters of the model. Let us start with the scalar spectrum. The adiabatic tilt is given by Eq. (10),

$$
n_{\text {ad }}=1-2 / N_{\text {hor }}=0.9692,
$$

for $N_{\text {hor }}=65$, while the spectral index for isocurvature fluctuations is

$$
n_{\text {iso }}=0.9722+4 \nu / 3 .
$$

Figure 5 shows some typical values of the parameter $\nu$ as a function of $M / m$, for different choices of $d_{10}$.

As for the tensor contribution, we considered:

$$
n_{T}=-1 / N_{\text {hor }}=-0.0154,
$$

and a tensor to scalar ratio given by:

$$
R=\frac{C_{2}^{T}}{C_{2}^{S}} \simeq-7 n_{T}=0.1077
$$

which is not negligible. In Fig. At the tensor contribution is very small and is already included in the solid line for

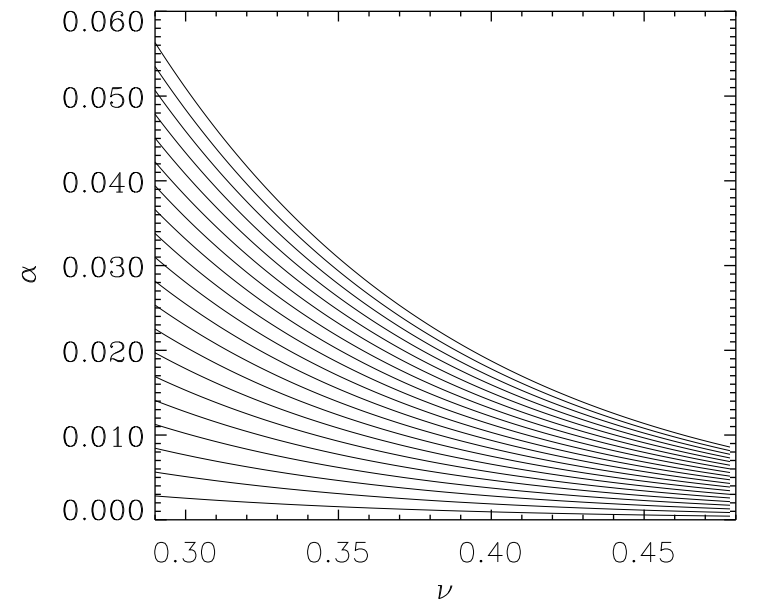

FIG. 6. Typical values of $\alpha$ as a function of the tilt parameter $\nu$ in the isocurvature spectrum. The different curves refer to different $d_{10}$ ranging from 1 to 20 , from bottom to top.

the adiabatic component, in order to make emphasis on its difference with respect to the isocurvature component.

With the above spectral properties, we determined the relative amplitude $\alpha$ in (16) to be related to the input parameters $d_{10}$ and $\nu$,

$$
\alpha=d_{10} \times 10^{-1.291-4.343 \nu} .
$$

For any value of $d_{10}$ between 1 and 10, the tilt parameter $\nu$ ranges between 0.29 and 0.48 . As a consequence, $n_{\text {iso }}$ is found to be between 1.36 and 1.61.3 Note that while $\nu$ fixes the value of the isocurvature spectral index, several $\alpha$ values are still possible, depending on the $d_{10}$ values. In fig. 6 we plot the value of $\alpha$ as a function of $\nu$, for $d_{10}$ in the range $1-20$. In any case, the values of $\alpha$ found are small, and for $d_{10}<32, \alpha$ never exceeds 0.09 . In the comparison with the data, we considered $\alpha<0.08$ and $1.36<n_{\text {iso }}<1.61$, and treated them as independent parameters, although they are not really independent [see Eq. (25)].

Note that we have represented in Fig. 6 the relative contribution $\alpha$ of the isocurvature component to the total power spectrum at COBE scales, i.e. large scales. Due to the strong positive tilt of the isocurvature spectrum $\left(n_{\text {iso }}>1\right)$, this relative contribution increases towards smaller scales. Since one of the observational constraints that we will consider in the following is represented by the number density of local galaxy clusters, it is interesting to estimate the isocurvature contribution at the characteristic cluster scales, $\sim 10 h^{-1} \mathrm{Mpc}$. To this purpose we

\footnotetext{
${ }^{3}$ Note that this is equivalent to $-2.64<n_{\text {iso }}<-2.39$ in the usual notation, where $n_{\text {iso }}=-3$ is the scale invariant isocurvature perturbation.
} 


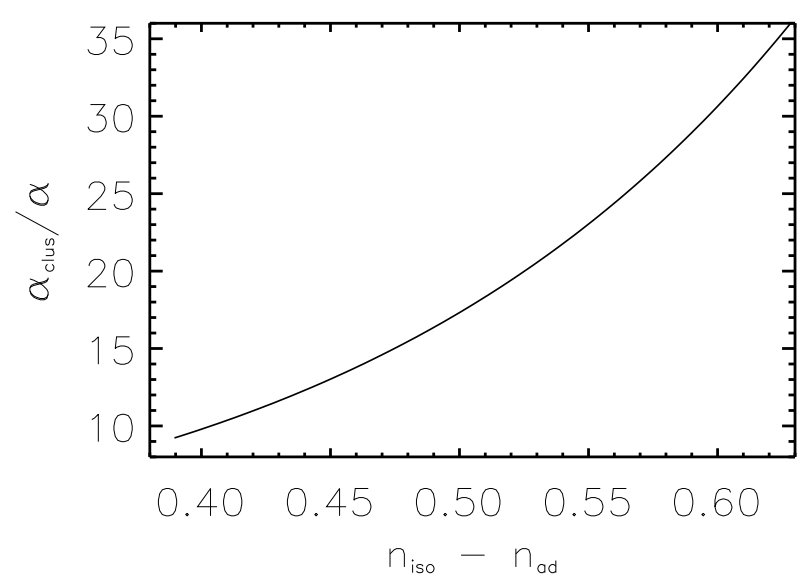

FIG. 7. The ratio $\alpha_{\text {clus }} / \alpha$ between the relative isocurvature contribution $\alpha$ at the scale of clusters and at COBE scales, as a function of the tilt difference, $n_{\text {iso }}-n_{\text {ad }}$.

introduce the quantity

$$
\alpha_{\text {clus }} / \alpha=\left(k_{\text {clus }} / k_{\text {hor }}\right)^{n_{\text {iso }}-n_{\text {ad }}}=(300)^{n_{\text {iso }}-n_{\text {ad }}},
$$

which provides the isocurvature contribution at the scale of galaxy clusters. We plot the cluster- to large-scale ratio of the isocurvature fraction in Fig. 7. It is apparent that the enhancement of the isocurvature contribution can be rather large, such that its effect is not negligible at the cluster scale, even for a rather small large-scale contribution.

We will discuss in Section $\mathrm{V}$ the implications of our results on the Linde-Mukhanov model. As for the cosmological parameters $\Omega_{m}, \Omega_{\Lambda}$ and $H_{0}$, we always considered flat models $\left(\Omega_{m}+\Omega_{\Lambda}=1\right)$, with $0.2 \leq \Omega_{m} \leq 1$, and $H_{0} \equiv 100 h=50,65,80 \mathrm{~km} \mathrm{~s}^{-1} \mathrm{Mpc}^{-1}$. As for the density parameter contributed by baryons, we take the value $\Omega_{\mathrm{B}}=0.019 h^{-2}$, which follows from the low deuterium abundance, as determined by Burles \& Tytler [28].

\section{OBSERVATIONAL CONSTRAINTS}

In this section we test our model against the available microwave background and large scale structure data, and we make predictions on the precision with which future satellite experiments will determine the relevant parameters. To this purpose, we use the Fisher matrix technique to determine how well MAP and Planck satellites will constrain the isocurvature contribution to the total power spectrum and its spectral index.

\section{A. The microwave background data}

In the comparison of the CMB data with the model predictions, we performed a $\chi^{2}$ analysis, first applied to
CMB data by Lineweaver et al. 29]. More precisely, we computed the $\chi^{2}$, as a function of the cosmological parameters $\vec{\lambda}$, on the band-power estimates of the CMB data, $\delta T^{\mathrm{obs}}(n)$, and the model predictions, $\delta T^{\bmod }(\vec{\lambda}, n)$, given $N_{\text {exp }}$ observed data points with their errors $\sigma$ :

$$
\chi^{2}(\vec{\lambda})=\sum_{n=1}^{N_{\text {exp }}}\left[\frac{\delta T^{\mathrm{obs}}(n)-\delta T^{\bmod }(\vec{\lambda}, n)}{\sigma(n)}\right]^{2} .
$$

Evaluating the expression above, we used the 41 data points 30] listed in table If, and the corresponding window functions. We chose not to introduce the recent points from MAT [31] and Python V 32] experiments because the former does not yet include calibration errors and the results of the latter are still under discussion. The value of $\chi^{2}$ obtained is a function of the model parameters $\vec{\lambda}$.

For each choice of the parameters $\Omega_{m}$ and $H_{0}$, which describe the Friedmann background, we compute the $\chi^{2}$ between model and data in the $\left(n_{\text {iso }}, \alpha\right)$ parameter space. In Table II we report the values of the parameters of the best fit, the corresponding $\chi_{\min }^{2}$ value, and the probability of getting that $\chi^{2}$ value with present data if the model considered is the real one.

For $h=0.5$ a small contribution of the isocurvature component is desirable, especially for low $\Omega_{m}$ universes. On the other hand, for $h=0.8$ the addition of an isocurvature contribution to the adiabatic mode doesn't provide a better fit to the data. In general, however, the allowed isocurvature fraction tends to be small $(\alpha<0.01)$, while the isocurvature spectral index is not significantly constrained (see Fig. (4). In any case, the best fit to the data is provided by the lowest $n_{\text {iso }}$ considered.

\section{B. Combining CMB and LSS constraints}

In order to further constrain the parameter space of allowed models, we will consider in this Section the constraints coming from large scale structure observations. In particular, we will constrain the shape of the power spectrum, by comparing to results from the analysis of galaxy clustering, and its amplitude by resorting to constraints from the local abundance of rich galaxy clusters.

As for the shape of the galaxy power spectrum, different determinations have been realized in the last few years, both for projected [26] and redshift [33] samples. Such analyses converge to indicate that the observed galaxy power spectrum is well reproduced by an adiabatic CDM-like $P(k)$, in a flat universe, with shape parameter $0.2 \lesssim \Gamma \lesssim 0.3$, for a scale invariant primordial spectrum [34].

According to the results of the previous section on the CMB constraints, the relative contribution of the isocurvature component of the fluctuations is always rather small. As a result, the shape of the purely adiabatic 
spectrum is never significantly changed by the isocurvature component. For this reason, in order to implement the constraint from the shape of the galaxy power spectrum, we will simply require in the following that the adiabatic component of our mixed fluctuation spectrum has a shape parameter $\Gamma$ lying in the $0.2-0.3$ range.

As for the amplitude of the power spectrum, a powerful constraint is represented by the number density of nearby galaxy clusters. Since rich galaxy clusters involve a typical mass of the order of $10^{15} h^{-1} M_{\odot}$, their number density is connected to the amplitude of density fluctuations at scales of about $10 h^{-1} \mathrm{Mpc}$. Analytical approaches, based on the method originally devised by Press \& Schechter [35], show that the cluster abundance actually constrains the quantity $\tilde{\sigma}_{8}=\sigma_{8} \Omega_{m}^{\beta}$, where $\sigma_{8}$ is the r.m.s. fluctuation amplitude within a top-hat sphere of $8 h^{-1} \mathrm{Mpc}$ and $\beta \simeq 0.4-0.5$, almost independent of the shape of the power spectrum and of the presence of a cosmological constant term 36].

Different analyses, based on the distributions of $X-$ ray cluster luminosities, $X$-ray temperatures and velocity dispersions of member galaxies, converge to values of $\tilde{\sigma}_{8}$ in the range $0.5-0.6$ 37. For definiteness, in the following we will use for our analysis the expression

$$
\sigma_{8}=(0.55 \pm 0.05) \Omega_{m}^{-0.43+0.09 \Omega_{m}}
$$

where the errors are intended to formally correspond to a $90 \%$ confidence level, while the $\Omega_{m}$ dependence is that provided by Girardi et al. [38] for a flat Universe, with $\Omega_{\Lambda}=1-\Omega_{m}$.

We show in Figure 8 constraints on the $n_{\text {iso }}-\alpha$ parameter space by combining results from LSS and CMB data. Each panel corresponds to a choice for the $\left(\Omega_{m}, h\right)$ parameters, among those reported in Table II, which agrees with the measured shape of the galaxy power spectrum.

As for the constraints from the abundance of local clusters, the $90 \%$ confidence level region [see Eq. (28)] is shown by the shaded area. We note that, as $n_{\text {iso }}$ increases, the contribution of the isocurvature fluctuations at the cluster scale also increases, thus requiring a smaller $\alpha$ value to keep the power spectrum amplitude at the level required by the cluster number density.

In order to establish confidence levels for model exclusion from the analysis of CMB anisotropies we consider the quantity $\Delta \chi^{2}=\chi^{2}-\chi_{\min }^{2}$, where $\chi_{\min }^{2}$ is the minimum value as reported in Table II. We assume the $\chi^{2}$ statistics for $N_{\text {dof }}=38$ to be normally distributed with mean $N_{\text {dof }}$ and r.m.s. scatter given by $\sqrt{2 N_{\text {dof }}}$. Accordingly, all the models displayed in Figure 8 correspond to an acceptable value of $\chi_{\min }^{2}$. The solid curve indicates the $90 \%$ c.l. upper limit on $\alpha$, which corresponds to $\Delta \chi^{2}=4.61$ for two significant fitting parameters.

As a general result, it is interesting to note that large scale structure constraints significantly contribute to further restrict the range of the allowed parameter space. For instance, the two models with $\left(\Omega_{m}, h\right)=(0.5,0.65)$ and $(0.4,0.80)$ are now ruled out, since they can not satisfy at the same time both the $\mathrm{CMB}$ and local cluster abundance constraints, while the model with $\left(\Omega_{m}, h\right)=$ $(0.5,0.50)$ is constrained by the cluster abundance to have $\alpha \lesssim 0.0015$.

It is worth reminding here that the constraint of Eq. (28) from the local cluster abundance has been derived in the literature under the assumption of Gaussian statistics for the density fluctuations. On the other hand, the density fluctuations predicted by our model are given by a scale dependent superposition of a Gaussian adiabatic components and of a non-Gaussian isocurvature component, whose probability density function (PDF) corresponds to a $\chi^{2}$ model with one degree of freedom. Although the extension of the Press-Schechter formalism to non-Gaussian statistics has been pursued by different authors [39], such attempts concentrated on scaleindependent PDF models. In our case, we expect that the positive skewness of the $\chi^{2}$-distribution should ease the formation of galaxy clusters, for a fixed $\tilde{\sigma}_{8}$, as a consequence of the broader high density tail in the PDF for the isocurvature component. Therefore, the net effect would go in the direction of decreasing the required fluctuation amplitude at the cluster scale and, thus, to somewhat increase the allowed $\alpha$ values. In any case, since the isocurvature component is always constrained to be relatively small even at the cluster scales $\left(\alpha_{\text {clus }} \lesssim 0.15\right.$; cf. Fig. 7), we are confident that our assumption of Gaussian statistics should be a sensible one.

\section{The future CMB experiments}

In this section we compute the estimates of the errors with which the future satellite experiments MAP and Planck will determine the isocurvature contribution to the CMB power spectrum. The aim here is to verify whether the future CMB data alone will be able to constrain small isocurvature contributions when other cosmological parameters are constrained at the same time.

In order to provide such an estimate, we resort to the Fisher information matrix approach [40]. When no polarization is considered, the Fisher information matrix is defined as

$$
\begin{aligned}
F_{i j} & =\sum_{l} \frac{\partial C_{l}}{\partial \lambda_{i}} \operatorname{Cov}^{-1} \frac{\partial C_{l}}{\partial \lambda_{j}}, \\
C o v & =\frac{2}{(2 l+1) f_{\mathrm{sky}}}\left(C_{l}+w^{-1} e^{l^{2} \sigma_{b}^{2}}\right)^{2} .
\end{aligned}
$$

Here $f_{\text {sky }}$ is the fraction of sky covered, $\sigma_{b}$ is the Gaussian beamwidth $\left(\sigma_{b}=\theta_{\text {fwhm }} / \sqrt{8 \ln 2}\right.$, and $\theta_{\text {fwhm }}$ is the full width at half maximum), $\lambda_{i}$ is the set of parameter whose errors have to be determined, and $w$ contains the information on the detector resolution and sensitivity:

$$
w=\frac{\sigma_{\text {pixel }}^{2} \Omega_{\text {pixel }}}{T_{0}^{2}},
$$

where $\Omega_{\text {pixel }} \simeq \theta_{\text {fwhm }}^{2}$ 


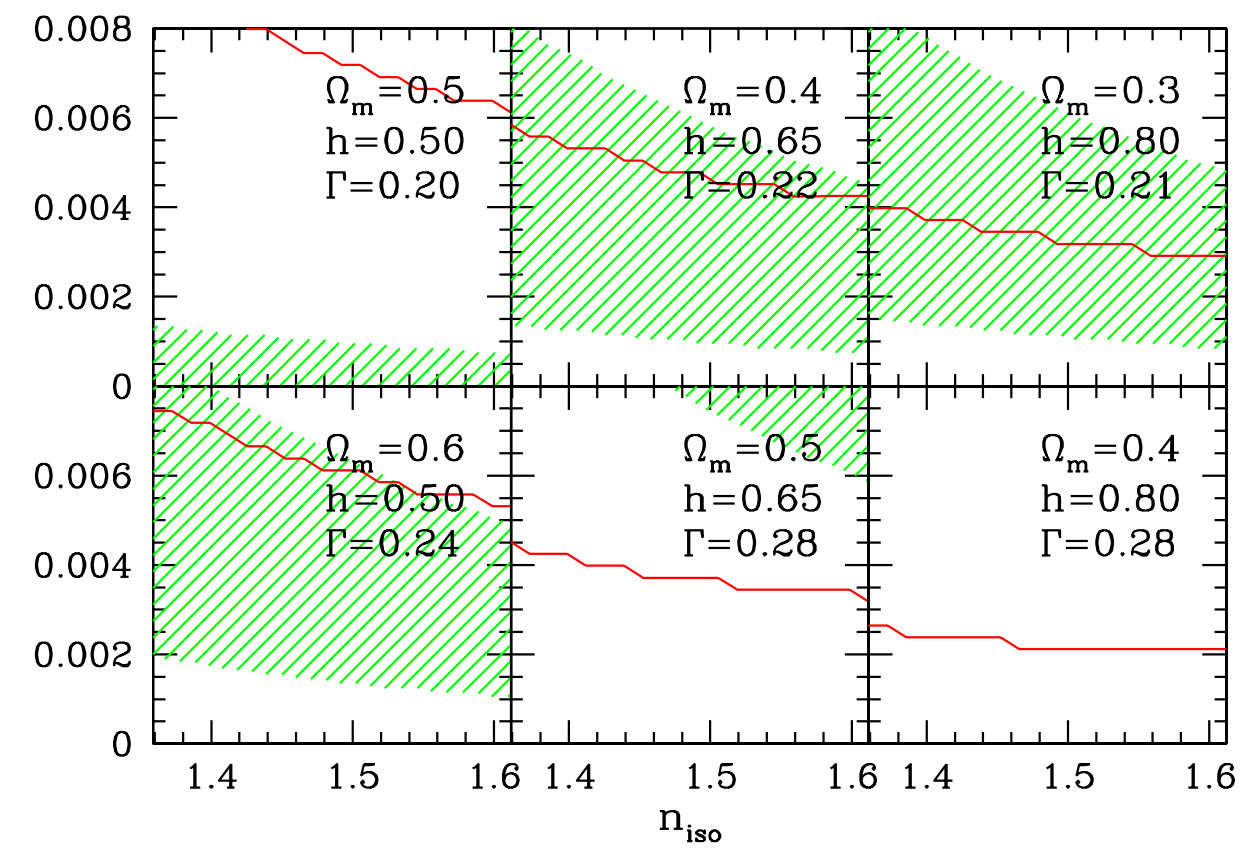

FIG. 8. Constraints on the $n_{\text {iso }}-\alpha$ plane from cluster abundance and CMB anisotropies. Each panel corresponds to a choice for the $\left(\Omega_{m}, h\right)$ parameters which satisfies the constraints on the shape parameter $\Gamma$. The corresponding $\Gamma$ values for each model are also reported. The shaded area correspond to the $90 \%$ c.l. from the cluster abundance, obtained according to eq.(28). The solid curves indicate the $90 \%$ c.l. upper limit on $\alpha$ from the CMB constraints (see text); oscillations in the shape of these curves are due to limitations in the numerical precision.

In the case that also polarization is considered, the expression for the Fisher and covariance matrices become more complicated, and we refer here to Ref. 41] for the explicit expression. We note that in the expression above a perfect foreground subtraction is assumed, so that the estimates found should be considered somehow ideal. Also notice that the expression reported here strictly holds for Gaussian perturbations. Therefore, its application to the Linde-Mukhanov model should be taken with some caution, especially in the very large multipole regime (i.e., small scales), where the non-Gaussian isocurvature contribution may be non negligible.

We estimate the expected errors on $\alpha$ and $n_{\text {iso }}$ with and without polarization. In the calculation of the Fisher matrix, we considered as free parameters the Hubble constant $h$, the baryon abundance $\Omega_{\mathrm{B}} h^{2}$, the normalization $C_{10}$, the reionization optical depth $\tau$, the isocurvature/adiabatic ratio $\alpha$, and the isocurvature spectral in-

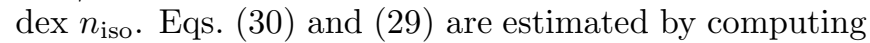
$C_{l}$ for a fiducial model with $\Omega_{m}=0.3$ (fixed), $h=0.65$, $\Omega_{\mathrm{B}} h^{2}=0.019, C_{10}=C_{10_{\mathrm{COBE}}}$ and $\tau=0.05$.

The precision in the determination of $\alpha$ and $n_{\text {iso }}$ is reported in table IV for different fiducial values, and different experiments. For both experiments, we combine different channels in order to give the estimates on the errors. More precisely, we consider the 3 highest frequency channels for MAP $(40,60,90 \mathrm{GHz})$, and the 5 central frequency channels from Planck $(70,100 \mathrm{GHz}$ channels from LFI and the 100, 143, $217 \mathrm{GHz}$ channels from HFI). The values of the beamwidths and sensitivities for the two experiments are reported in table III, according to Refs. [1.2].

As a result, we find that the future satellite experiments will be able to constrain the spectral index $n_{\text {iso }}$ much better than the present data. Clearly, $n_{\text {iso }}$ is better determined if a higher isocurvature component is allowed, and for a given $\alpha$ a higher fiducial isocurvature anti-tilt ensures a smaller uncertainty in the parameter determination. Considering polarization is also useful in this respect, and typically reduces the errors by almost a factor of 2 . If the isocurvature contribution is as low as indicated by our present analysis, $n_{\text {iso }}$ will be anyway determined by Planck with an error of about 0.1.

As for the constraints on $\alpha$, table IV shows that if the isocurvature contribution is quite large $(\alpha \simeq 0.015)$ both MAP and Planck will be able to detect it; on the contrary, if $\alpha$ is as low as the present data seem to suggest, only Planck (with polarization information included) will be able to claim a detection, while MAP will only put an upper limit at the $0.002-0.003$ level. Note that the use of polarization data certainly helps in reducing the error, although we don't find the big improvement claimed by [18]. This may occur because we keep the scalar to tensor ratio $R$ fixed in this model, and therefore we are not affected by the degeneracy of this parameter with $\alpha$. Polarization is useful in breaking the degeneracy between the reionization parameter $\tau$ and the isocurvature contribution $\alpha$ and helps in reducing the errors especially in 
the case of the Planck experiment.

\section{DISCUSSION}

In this paper we have investigated the consequences of a CDM cosmology with mixed isocurvature and adiabatic initial conditions as prescribed by a generic LindeMukhanov inflationary model.

We showed how the total spectra are modified by the isocurvature contribution, as both the primordial spectral index for the isocurvature power spectrum, $n_{\text {iso }}$, and the isocurvature fraction, $\alpha$, are varied within their allowed ranges. In order to constrain the parameters of the model we resorted to available data on the CMB anisotropy, as well as on the large-scale structure constraints from the shape of the galaxy power spectrum and the number density of nearby galaxy clusters. Observational constraints from CMB and LSS data have been shown to provide complementary informations. As a result, we found that the allowed isocurvature contribution at COBE scales is always very small, $\alpha \lesssim 0.006$. Therefore, our results generalizes to the $n_{\text {iso }}>1$ case and strengthen the conclusions reached in Ref. [16] based on LSS constraints alone.

We note that, even allowing for the strong positive tilt of the isocurvature component, the permitted isocurvature contribution at the cluster scales is always small, with $\alpha_{\text {clus }} \lesssim 0.15$. However, this contribution is expected to increase to $\sim 50 \%$ level when we consider smaller scales, $\lesssim 1 h^{-1} \mathrm{Mpc}$, which are relevant for galaxy formation. Therefore, the resulting $\chi^{2}$ (positive skewness) non-Gaussian statistics contributed by the isocurvature fluctuations can play a significant role to ease the galaxy formation at high redshift. The phenomenological implications on galaxy formation of a small isocurvature contribution at the COBE scales remains to be investigated in detail.

As for the determination of the isocurvature spectral index $n_{\text {iso }}$, the best fit to the CMB data is always provided by the lowest $n_{\text {iso }}$ considered; although the limits from LSS and CMB data in Fig. 8 show a mild dependence on $n_{\text {iso }}$. In this respect, future CMB experiments can help to set more accurate limits on $n_{\text {iso }}$, with an optimistic estimate of $3 \%$ and $1 \%$ (high $n_{\text {iso }}$ and $\alpha$ ) and a more realistic one of $20 \%$ and $8 \%$ for MAP and Planck respectively.

Finally, we showed that only the Planck experiment will be sensitive enough to detect a possible non vanishing value for the $\alpha$ parameter within the limits already set by present CMB and LSS data.

\section{ACKNOWLEDGEMENTS}

J.G.B. is supported by the Royal Society of London. E.P. is a CITA national fellow. The authors wish to ac- knowledge SISSA for hosting all of them during different phases of this work. The authors thank Andrei Linde, Andrew Liddle, and Douglas Scott for generous discussions.

[1] http://www.map.gsfc.nasa.gov

[2] http://wWw.astro.estec.esa.nl/SA-general/ Projects/Planck/

[3] See, for example:

J.R. Bond, G. Efstathiou and M. Tegmark, M.N.R.A.S. 291 (1997) L33 [astro-ph/9702100]; L. Knox, Phys. Rev. D 52 (1995) 4307 [astro-ph/9504054]; G. Jungman, M. Kamionkowski, A. Kosowsky and D. Spergel, Phys. Rev. Lett. 76 (1996) 1007 astro-ph/9507080; M. Zaldarriaga, D.N. Spergel and U. Seljak, Ap. J. 488 (1997) 1 astro-ph/9702157 and references therein.

[4] A.D. Linde, Phys. Lett. B 158 (1985) 375; L.A. Kofman, Phys. Lett. B 173 (1985) 400; A.D. Linde and L.A. Kofman, Nucl. Phys. B 282 (1987) 555.

[5] G. Efstathiou and J.R. Bond, M.N.R.A.S. 218 (1986) 103.

[6] H. Kodama and M. Sasaki, Int. J. Mod. Phys. A 2 (1987) 491.

[7] W. Hu and N. Sugiyama, Phys. Rev. D 51 (1995) 2599 astro-ph/9411008.

[8] See, for example: D.S. Salopek, Phys. Rev. D 45 (1992) 1139; L. Kofman and A.D. Linde, Nucl. Phys. B 282 (1987) 555; L. Kofman, A.D. Linde and J. Einasto, $\mathrm{Na}$ ture 326 (1987) 48; L. Kofman, Phys. Lett. B 173 (1986) 400; M. Bucher and Y. Zhu, Phys. Rev. D 55 (1997) 7415 astro-ph/9610223.

[9] P.J.E. Peebles, Nature 327 (1987) 210; Ap. J. 315 (1987) L73; J.R. Bond and G. Efstathiou, M.N.R.A.S. 22 (1987) 33; K. Gorski and J. Silk, Ap. J. 346 (1989) 1; J. Yokoyama and Y. Suto, Ap. J. 379 (1991) 427; T. Suginohara and Y. Suto, Ap. J. 387 (1992) 431; R. Cen, J.P. Ostriker and P.J.E. Peebles, Ap. J. 415 (1993) 423; T. Chiba, N. Sugiyama and Y. Suto, Ap. J. 429 (1994) 427 astro-ph/9311012.

[10] W. Hu, E. Bunn and N. Sugiyama, Ap. J. 447 (1995) 59 astro-ph/9501034.

[11] J.A. Frieman and E. Gaztañaga, Ap. J. 521 (1999) L83 astro-ph/9903423.

[12] P.J.E. Peebles, Ap. J. 510 (1999) 523; Ap. J. 510 (1999) 531.

[13] M. Bucher, K. Moodley and N. Turok, The general primordial cosmic perturbation, astro-ph/9904231.

[14] S. Mollerach and S. Matarrese, Phys. Rev. D 45 (1992) 1961; N. Deruelle, C. Gundlach and D. Langlois, Phys. Rev. D 46 (1992) 5337; A.A. Starobinsky and J. Yokoyama, Density fluctuations in Brans-Dicke inflation, gr-qc/9502002 J. García-Bellido and D. Wands, Phys. Rev. D 52 (1995) 6739 gr-qc/9506050; M. Sasaki and E.D. Stewart, Prog. Theor. Phys. 95 (1996) 71 astro-ph/9507001; J. García-Bellido and D. Wands, 
Phys. Rev. D 53 (1996) 5437 astro-ph/9511029; D. Langlois, Correlated adiabatic and isocurvature perturbations from double inflation, astro-ph/9906080.

[15] R. Stompor, A.J. Banday and K.M. Gorski, Ap. J. 463 (1996) 8 astro-ph/9511087.

[16] M. Kawasaki, N. Sugiyama and T. Yanagida, Phys. Rev. D 54 (1996) 2442 hep-ph/9512368; T. Kanazawa, M. Kawasaki, N. Sugiyama and T. Yanagida, Prog. Theor. Phys. 102 (1999) 71 astro-ph/9805102.

[17] A.D. Linde and V. Mukhanov, Phys. Rev. D 56 (1997) 535 astro-ph/9610219.

[18] K. Enqvist and H. Kurki-Suonio, Constraining isocurvature fluctuations with the Planck Surveyor, astro-ph/9907221.

[19] U. Seljak and M. Zaldarriaga, Ap. J. 469 (1996) 437 astro-ph/9603033.

[20] CMBFAST webpage: http://www.sns.ias.edu $\sim_{\text {matiasz/CMBFAST/cmbfast.html }}$

[21] C.L. Bennett et al., Ap. J. 464 (1996) L1 astro-ph/9601067.

[22] J.R. Bond, in Cosmology and Large Scale Structure, Les Houches Summer School Course LX, R. Schaeffer ed., Elsevier Science Press, Amsterdam, 1996.

[23] E.F. Bunn and M. White, Ap. J. 480 (1997) 6 astro-ph/9706060.

[24] J.M. Bardeen, J.R. Bond, N. Kaiser and A.S. Szalay, Ap. J. 304 (1986) 15.

[25] N. Sugiyama, Ap. J. Suppl. $100 \quad$ (1995) 281 astro-ph/9412025.

[26] C.M. Baugh and G. Efstathiou, M.N.R.A.S. 265 (1993) 145; E. Gaztañaga and C.M. Baugh, M.N.R.A.S. 294 (1998) 229 astro-ph/9704246.

[27] J.R. Bond, A.H. Jaffe, L. Knox, Radical compression of cosmic microwave background data astro-ph/9808264.

[28] S. Burles and D. Tytler, Ap. J. $\mathbf{5 0 7}$ (1998) 732 astro-ph/9712109.

[29] C.H. Lineweaver, D. Barbosa, A. Blanchard and J.G. Bartlett, A. and A., 322 (1997) 365 astro-ph/9610133.

[30] M. Tegmark and A. Hamilton, Uncorrelated measurements of the CMB power spectrum, astro-ph/9702019; G.S. Tucker, H.P. Gush, M. Halpern, I. Shinkoda, $A p$. J. 475 (1997) L73; K. Ganga, L. Page, E. S. Cheng, S. Meyer, Ap. J. 432 (1994) L15; S.T. Tanaka et al., Ap. J. 468 (1996) L81; P. de Bernardis et al., Ap. J. 422 (1994) L33; A. de Oliveira-Costa et al., Ap. J. 509 (1998) L77; S. Masi et al., Ap. J. 463 (1996) L47; C.B. Netterfield et al., Ap. J. 474 (1997) 47; S.T. Tanaka et al., Ap. J. 468 (1996) L81; E. Leitch et al., A measurement of anisotropy in the cosmic microwave background on 7-22 arcminute scales, astro-ph/9807312 (1998); J.C. Baker et al., Detection of cosmic microwave background structure in a second field with the Cosmic Anisotropy Telescope, astro-ph/9904415; J.O. Gundersen et al., Ap. J. 433 (1995) L57; B. Femenia, R. Rebolo, C.M. Gutierrez, M. Limon, L. Piccirillo, Ap. J. 498 (1998) 117; S. Hancock et al., M.N.R.A.S. 289 (1997) 505; L. Piccirillo and P. Calisse, Ap. J. 411 (1993) 529; J.O. Gundersen et al., Ap. J. 433 (1995) L57; S.R. Platt et al., Ap. J. 475 (1997) L1; P.F.S. Scott et al., Ap. J. 461 (1996) L1; E.S. Cheng et al., Ap. J. 456 (1996) L71; E.S. Cheng et al.,
Ap. J. 488 (1997) L59; S.T. Tanaka et al., Ap. J. 468 (1996) L81.

[31] E. Torbet et al., Ap. J. $\quad \mathbf{5 2 1} \quad$ (1999) $\quad$ L79 astro-ph/9905100.

[32] K. Coble et al., Anisotropy in the cosmic microwave background at degree angular scales: Python $V$ results, astro-ph/9902195.

[33] H. Lin et al., Ap. J. 471 (1996) 617 [astro-ph/9606055]; H. Tadros et al., M.N.R.A.S. $\mathbf{3 0 5}$ (1999) 527 astro-ph/9901351.

[34] J.A. Peacock and S.J. Dodds, M.N.R.A.S. 267 (1994) 1020 astro-ph/9311057; A.R. Liddle, D.H. Lyth, R.H. Schaefer, Q. Shafi and P.T.P. Viana, M.N.R.A.S. 281 (1996) 531 astro-ph/9511057.

[35] W.H. Press and P. Schechter, Ap. J. 187 (1974) 425; C.G. Lacey and S. Cole, M.N.R.A.S. 271 (1994) 676 astro-ph/9402069.

[36] S.D.M. White, G. Efstathiou and C.S. Frenk, M.N.R.A.S. 262 (1993) 1023.

[37] V.R. Eke, S. Cole and C.S. Frenk, M.N.R.A.S. 282 (1996) 263 astro-ph/9601088; T. Kitayama and Y. Suto, Ap. J. 469 (1996) 480 astro-ph/9704141; M. Markevitch, Ap. J. 504 (1998) 27; S. Borgani, P. Rosati, P. Tozzi and C. Norman, Ap. J. 517 (1999) 40 astro-ph/9901017; P.T.P. Viana and A.R. Liddle, M.N.R.A.S. 303 (1999) 535 astro-ph/9803244.

[38] M. Girardi, S. Borgani, G. Giuricin, F. Mardirossian and M. Mezzetti, Ap. J. 506 (1998) 45 astro-ph/9804188.

[39] S. Colafrancesco, F. Lucchin and S. Matarrese, Ap. J. 345 (1989) 3; W.A. Chiu, J.P. Ostriker and M.A. Strauss, Ap. J. 494 (1998) 479 astro-ph/9708250; J. Robinson and J.E. Baker, Evolution of the cluster abundance in non-gaussian models, astro-ph/9905098; K. Koyama, J. Soda and A. Taruya, Constraints on a non-gaussian $\left(\chi_{m}^{2}\right)$ CDM model, astro-ph/9903027.

[40] M. Tegmark, A. Taylor and A. Heavens, Ap. J. 480 (1997) 22 astro-ph/9603021.

[41] M. Zaldarriaga, D.N. Spergel, U. Seljak, Ap. J. 488 (1997) 1 astro-ph/9702157. 


\begin{tabular}{|c|c|c|c|}
\hline Experiment & $\Delta T_{l} \pm \sigma(\mu K)$ & $l_{\text {eff }}$ & reference \\
\hline COBE & $8.5_{-8.5}^{+16}$ & 2.1 & Tegmark \& Hamilton (1997) \\
\hline COBE & $28.0_{-10.4}^{+7.5}$ & 3.1 & Tegmark \& Hamilton (1997) \\
\hline COBE & $34.0_{-7.2}^{+5.9}$ & 4.1 & Tegmark \& Hamilton (1997) \\
\hline $\mathrm{COBE}$ & $25.1_{-6.6}^{+5.2}$ & 5.6 & Tegmark \& Hamilton (1997) \\
\hline $\mathrm{COBE}$ & $29.4_{-4.1}^{+3.6}$ & 8.0 & Tegmark \& Hamilton (1997) \\
\hline COBE & $27.7_{-4.5}^{+3.9}$ & 10.9 & Tegmark \& Hamilton (1997) \\
\hline COBE & $26.1_{5.3}^{+4.4}$ & 14.3 & Tegmark \& Hamilton (1997) \\
\hline COBE & $33.0_{-5.4}^{+4.6}$ & 19.4 & Tegmark \& Hamilton (1997) \\
\hline SASK & $49.0_{-5.0}^{+8.4}$ & 86 & Netterfield et al (1997) \\
\hline SASK & $69.0_{-6.0}^{+7.0}$ & 166 & Netterfield et al (1997) \\
\hline SASK & $85.0_{-8.0}^{+10.0}$ & 236 & Netterfield et al (1997) \\
\hline SASK & $86.0_{-10.0}^{+12.0}$ & 285 & Netterfield et al (1997) \\
\hline SASK & $69.0_{28.0}^{+19.0}$ & 348 & Netterfield et al (1997) \\
\hline CAT & $50.8_{-15.4}^{+15.4}$ & 396 & Scott et al (1996) \\
\hline CAT & $49.0_{-16.9}^{+16.9}$ & 608 & Scott et al (1996) \\
\hline CAT & $57.3_{-13.6}^{+10.9}$ & 415 & Baker et al (1998) \\
\hline FIRS & $29.4_{-7.7}^{+7.8}$ & 10 & Ganga et al (1994) \\
\hline TENERIFE & $34.1_{-12.5}^{+12.5}$ & 20 & Hancock et al (1997) \\
\hline SP91 & $30.2_{-5.5}^{+8.9}$ & 57 & Gundersen et al (1995) \\
\hline SP94 & $36.3_{-6.1}^{+13.6}$ & 57 & Gundersen et al (1995) \\
\hline BAM & $48.4_{-16.5}^{+16.5}$ & 74 & Tucker et al (1997) \\
\hline ARGO & $39.1_{-8.7}^{+8.7}$ & 95 & de Bernardis et al (1994) \\
\hline ARGO & $46.8_{-12.1}^{+9.5}$ & 95 & Masi et al (1996) \\
\hline MAX-GUM & $54.5_{-10.9}^{+16.4}$ & 145 & Tanaka et al (1996) \\
\hline MAX-ID & $46.3_{-13.6}^{+21.8}$ & 145 & Tanaka et al (1996) \\
\hline MAX-SH & $49.1_{-16.4}^{+21.8}$ & 145 & Tanaka et al (1996) \\
\hline MAX-HR & $32.7_{-8.2}^{+10.4}$ & 145 & Tanaka et al (1996) \\
\hline MAX-PH & $51.8_{-10.9}^{+19.1}$ & 145 & Tanaka et al (1996) \\
\hline PYTHON1 & $54.0_{-12.0}^{+14.0}$ & 92 & Platt et al (1996) \\
\hline PYTHON2 & $58.0_{-13.0}^{+15.0}$ & 177 & Platt et al (1996) \\
\hline IAC & $112.0_{-60.0}^{+65.0}$ & 33 & Femenia et al (1998) \\
\hline IAC & $55.0_{-22.0}^{+27.0}$ & 53 & Femenia et al (1998) \\
\hline IAB & $94.5_{-41.8}^{+41.8}$ & 125 & Piccirillo \& Calisse (93) \\
\hline MSAM & $62.0_{-21.7}^{+21.0}$ & 143 & Cheng et al (1996) \\
\hline MSAM & $60.4_{-20.1}^{+20.1}$ & 249 & Cheng et al (1996) \\
\hline MSAM & $50.0_{-11.0}^{+16.0}$ & 160 & Cheng et al (1997) \\
\hline MSAM & $65.0_{-13.0}^{+18.0}$ & 270 & Cheng et al (1997) \\
\hline QMAP & $47.0_{-7}^{+6}$ & 80 & deOliveira-Costa et al (98) \\
\hline QMAP & $59.0_{-7}^{+6}$ & 126 & deOliveira-Costa et al (98) \\
\hline QMAP & $52.0_{-5}^{+5}$ & 111 & deOliveira-Costa et al (98) \\
\hline OVRO & $56.0_{-6.6}^{+8.5}$ & 589 & Leitch et al (1998) \\
\hline
\end{tabular}

TABLE I. Data points used in the $\chi^{2}$ analysis. First column is the experiment, second column is the experimental value with its error, third column is the effective multipole number, and fourth column is the reference paper.

\begin{tabular}{ccccc}
\hline \hline$\Omega_{m}$ & $n_{\text {iso }}$ & $\alpha$ & $\chi_{\min }$ & $P\left(\chi<\chi_{\min }\right)$ \\
\hline 0.2 & 1.359 & 0.006 & 21.6 & 0.015 \\
0.3 & 1.359 & 0.003 & 21.9 & 0.017 \\
0.4 & 1.359 & 0.001 & 22.7 & 0.023 \\
0.5 & 1.359 & 0 & 23.5 & 0.032 \\
0.6 & 1.359 & 0 & 24.8 & 0.048 \\
0.7 & 1.359 & 0 & 26.4 & 0.078 \\
0.8 & 1.359 & 0 & 28.3 & 0.126 \\
0.9 & 1.359 & 0 & 30.5 & 0.200 \\
1 & 1.359 & 0 & 33.5 & 0.323 \\
\hline 0.2 & 1.359 & 0.001 & 24.4 & 0.043 \\
0.3 & 1.359 & 0 & 26.4 & 0.08 \\
0.4 & 1.359 & 0 & 29.2 & 0.15 \\
0.5 & 1.359 & 0 & 32.7 & 0.29 \\
0.6 & 1.359 & 0 & 37.4 & 0.50 \\
0.7 & 1.359 & 0 & 43.1 & 0.74 \\
0.8 & 1.359 & 0 & 49.2 & 0.89 \\
0.9 & 1.359 & 0 & 55.5 & 0.97 \\
1 & 1.359 & 0 & 61.8 & 0.99 \\
\hline 0.2 & 1.359 & 0 & 30.2 & 0.19 \\
0.3 & 1.359 & 0 & 36.0 & 0.44 \\
0.4 & 1.359 & 0 & 44.1 & 0.77 \\
0.5 & 1.359 & 0 & 52.8 & 0.94 \\
0.6 & 1.359 & 0 & 61.5 & 0.99 \\
0.7 & 1.359 & 0 & 69.8 & 0.99 \\
0.8 & 1.359 & 0 & 77.8 & 0.99 \\
0.9 & 1.359 & 0 & 85.4 & 0.99 \\
1 & 1.359 & 0 & 92.6 & 0.99 \\
\hline \hline & & 0 & & \\
\hline
\end{tabular}

TABLE II. Results of the $\chi^{2}$ analysis for different models ( $h=0.50,0.65,0.80$ from top to bottom). Column 1: the matter density parameter. Column 2 and 3: the best fit values for $n_{\text {iso }}$ and $\alpha$ respectively. Column 4: the corresponding $\chi_{\min }^{2}$ value. Column 5: the probability of getting a smaller $\chi^{2}$, assuming that this is the real model. We considered here 41 experimental points and 3 free parameters $\left(\alpha, n_{\text {iso }}\right.$, and the normalization), which corresponds to $N_{\text {dof }}=38$ degrees of freedom.

\begin{tabular}{ccccc}
\hline \hline Experiment & frequency $(\mathrm{GHz})$ & $\theta_{\mathrm{fwhm}}$ & $\sigma_{p}(\mu \mathrm{K})$ & $\sigma_{p}^{\text {pol }}(\mu \mathrm{K})$ \\
\hline MAP & 40 & $0.47^{\circ}$ & 35 & 49.3 \\
MAP & 60 & $0.35^{\circ}$ & 35 & 49.3 \\
MAP & 90 & $0.21^{\circ}$ & 35 & 49.3 \\
Planck-LFI & 70 & $14^{\prime}$ & 9.8 & 13.8 \\
Planck-LFI & 100 & $10^{\prime}$ & 11.72 & 16.5 \\
Planck-HFI & 100 & $10.7^{\prime}$ & 4.63 & - \\
Planck-HFI & 143 & $8.0^{\prime}$ & 5.45 & 10.2 \\
Planck-HFI & 217 & $5.5^{\prime}$ & 11.7 & 26.2 \\
\hline \hline
\end{tabular}

TABLE III. Beamwidths and sensitivities of the satellites experiments MAP and Planck. 


\begin{tabular}{cccccc}
\hline \hline $\begin{array}{c}\text { fiducial } \\
\alpha\end{array}$ & $\begin{array}{c}\text { fiducial } \\
n_{\text {iso }}\end{array}$ & $\begin{array}{c}\text { MAP } \\
\delta \alpha\end{array}$ & $\begin{array}{c}\text { MAP } \\
\delta n_{\text {iso }}\end{array}$ & $\begin{array}{c}\text { Planck } \\
\delta \alpha\end{array}$ & $\begin{array}{c}\text { Planck } \\
\delta n_{\text {iso }}\end{array}$ \\
\hline 0.002 & 1.439 & 0.0035 & 0.315 & 0.0024 & 0.209 \\
0.002 & 1.572 & 0.0022 & 0.195 & 0.0016 & 0.139 \\
0.008 & 1.439 & 0.0044 & 0.094 & 0.0031 & 0.064 \\
0.008 & 1.572 & 0.0030 & 0.062 & 0.0023 & 0.047 \\
0.015 & 1.439 & 0.0054 & 0.059 & 0.0040 & 0.042 \\
0.015 & 1.572 & 0.0039 & 0.041 & 0.0031 & 0.031 \\
\hline 0.002 & 1.439 & 0.0025 & 0.297 & 0.0013 & 0.126 \\
0.002 & 1.572 & 0.0015 & 0.172 & 0.0008 & 0.073 \\
0.008 & 1.439 & 0.0031 & 0.091 & 0.0017 & 0.039 \\
0.008 & 1.572 & 0.0020 & 0.056 & 0.0010 & 0.024 \\
0.015 & 1.439 & 0.0038 & 0.059 & 0.0020 & 0.025 \\
0.015 & 1.572 & 0.0026 & 0.038 & 0.0014 & 0.016 \\
\hline \hline
\end{tabular}

TABLE IV. Estimation of the isocurvature mode parameters with the future satellite experiments MAP and Planck. Top: polarization not considered. Bottom: polarization considered. The first two columns indicate the fiducial value considered, and the others indicate the estimated errors in the parameters. 